\title{
Editorial
}

\section{What is new with AF guidelines 2020?}

During the last European Society of Cardiology Congress in 2020, the new guidelines for atrial fibrillation (AF) diagnosis and management was released (1). We summarize here the key points from these International guidelines.

It is well established in this document that electrocardiogram (ECG) documentation is required to establish the diagnosis of AF. "Clinical AF" is defined as symptomatic or asymptomatic AF that is documented by surface ECG (at least $\geq 30$ seconds on ambulatory monitor, including wearable-recorded ECG or a 12-lead ECG). "Subclinical AF" refers to individuals without symptoms, or in whom pacemaker or implantable cardioverter-defibrillator (ICD) interrogation reveals "atrial high rate episode (AHRE)," and without AF detection on surface ECG.

While there is a significant amount of data on the management of "clinical AF," data on optimal management of AHRE and subclinical AF are lacking. Noteworthy, some terminology should be abandoned such as chronic AF, valvular and non-valvular AF, and lone $A F$, which may be confusing.

Actually, these last guidelines put the stress on the importance of managing cardiovascular risk factors and concomitant comorbidities. This strategy has been summarized as the $C C$ to $A B C$ strategy:

-Confirm AF

-Characterize AF (the 4S-AF scheme)

-Treat AF: The ABC Pathway

The 4S-AF scheme

Once diagnosed with AF, patients should undergo a "structured characterization," using the 4S-AF scheme. There are four components:

-Stroke risk evaluation using the traditional $\mathrm{CHA}_{2} \mathrm{DS}_{2-}$ VASc clinical stroke risk score

-Symptom severity assessment using the EHRA symptom score

-Severity of AF burden
-Substrate severity using clinical assessment and imaging

CHA2DS2-VASc clinical stroke risk score is used to identify "low risk" patients $\left(\mathrm{CHA}_{2} \mathrm{DS}_{2}\right.$-VASc score $=0$ in men, or 1 in women), who should not be offered antithrombotic therapy. Antiplatelet therapy alone is not recommended for stroke prevention in AF (Class III). Oral anticoagulation (OAC) is recommended for stroke prevention in AF patients with $\mathrm{CHA}_{2} \mathrm{DS}_{2}$-VASc score $\geq 2$ in men or $\geq 3$ in women, and it should be considered in patients with a $\mathrm{CHA}_{2} \mathrm{DS}_{2}$-VASc score of 1 in men or 2 in women, with treatment individualized based on net clinical benefit and patient preferences. Non-vitamin $\mathrm{K}$ oral anticoagulants (NOACs) are preferred over vitamin $\mathrm{K}$ antagonists (warfarin) (excluding patients with mechanical heart valves or moderate-to-severe mitral stenosis). In AF patients with acute coronary syndrome (ACS) undergoing an uncomplicated percutaneous coronary intervention, early cessation ( $\leq 1$ week) of aspirin and continuation of dual therapy with an OAC and a P2Y12 inhibitor (preferably clopidogrel) for up to 12 months is recommended (class I). Triple therapy with aspirin, clopidogrel, and an OAC for longer than 1 week after an ACS should be considered when risk of stent thrombosis outweighs the bleeding risk, with the total duration $\leq 1$ month.

In patients at risk for stroke with postoperative AF after noncardiac surgery, long-term OAC therapy to prevent thromboembolic events should be discussed, considering the anticipated net clinical benefit of OAC and informed patient choice. Beta-selective blockers should not be routinely used in order to prevent postoperative AF in patients undergoing noncardiac surgery (Class III).

Address for Correspondence: Sok-Sithikun Bun, Pasteur University Hospital, Nice, France, Phone: +33492037733, Fax: +37797989732, Email: sithi.bun@gmail.com

Received: 31.01.2021 Accepted: 01.02.2021 Copyright@ 2021 Heart, Vessels and Transplantation doi: $10.24969 /$ hvt.2020.243 
The $A B C$ Pathway can be summarized as following:

\section{-A: Anticoagulation/Avoid stroke}

-B: Better symptom control (rate and rhythm control, including catheter ablation for rhythm control)

-C: Cardiovascular risk factors and concomitant diseases (co-morbidities and lifestyle interventions)

Obesity and obstructive sleep apnea are major risk factors for AF. It has been shown that weight loss improves outcomes in patients with AF. Care should be taken for developing aggressive risk factor reduction programs focusing on weight management, hyperlipidemia, obstructive sleep apnea, hypertension, diabetes, smoking cessation, and alcohol intake reduction. They are all associated with a significant reduction of AF burden after ablation, if optimally managed.

The importance of AF screening for AF is emphasized in these guidelines, and opportunistic screening should be recommended in patients $\geq 65$ years old, hypertensive patients, and in patients with obstructive sleep apnea. Systematic ECG screening should be considered to detect $A F$ in patients after 75 years, or those at high risk of stroke.

Patient preferences should be considered, and an assessment of "patient-reported outcome" measures is recommended. The role of listening to the patient is central to these new guidelines. Integrated, patientcentered AF management may be accomplished through the coordination of a cardiologist, AF nurse, general practitioner, and pharmacist. New tools are introduced in order to measure quality of care and identify opportunities for improved treatment quality and AF patient outcome, which should be considered by practitioners and institutions.

Lenient rate control (heart rate $<110 \mathrm{bpm}$ on ECG) is often sufficient to improve AF-related symptoms. Rhythm control strategy is mainly indicated for reduction in AF-related symptoms and improvement of quality of life.

\section{AF Catheter ablation}

Catheter ablation is a well-established, safe, and superior alternative to antiarrhythmic drugs (AAD) for maintenance of sinus rhythm. While catheter ablation has not been shown until now to reduce total mortality or stroke in patients with AF and normal left ventricular function, it may be discussed in selected AF patients with heart failure (HF) with reduced ejection fraction to improve survival, and reduce HF hospitalizations (Class Ila). In patients with tachycardia-induced cardiomyopathy, catheter ablation reverses left ventricular dysfunction in most cases.

An evolution can be seen from the previous 2016 AF guidelines, because catheter ablation has now become a Class I indication in paroxysmal and persistent forms (even in the presence of major risk factors for recurrence), after one failed class I or III AAD; and a Class Ila recommendation after one failed beta-blocker treatment.

AF ablation can be proposed as a first-line treatment for rhythm control in selected patients with symptomatic paroxysmal AF (Class Ila), or with persistent AF without major risk factors for recurrence (Class IIb). Complete electrical isolation of the pulmonary veins is recommended during all $\mathbf{A F}$ catheter-ablation procedures (Class I), while additional left atrial ablation lesions are not yet well established (Class IIb). OAC should be continued for at least two months after the procedure, and extended beyond according to stroke risk assessment.

Finally, in recent years, substantial progress has been made in the detection of $\mathrm{AF}$ and its management, and new evidence is timely integrated in this third edition of the ESC guidelines on AF.

The Atrial Fibrillation Better Care (ABC) approach in the 2020 ESC AF Guidelines is in line with the previous approach introduced in the 2016 ESC AF Guidelines, with the goal to further improve the structured management of AF patients, promote patient values, and finally improve patient outcomes.

Sok-Sithikun Bun, Emile Ferrari Pasteur University Hospital, Cardiology Department, Nice, France

Peer-review: Internal Conflict of interest: None to declare Authorship: S-S.B. and E.F. are equally contributed to article preparation Acknowledgement and funding: None to declare 


\section{References}

1.Hindricks G, Potpara T, Dagres N, Arbelo E, Bax JJ, Blomström-Lundqvist C, et al; ESC Scientific Document Group. 2020 ESC Guidelines for the diagnosis and

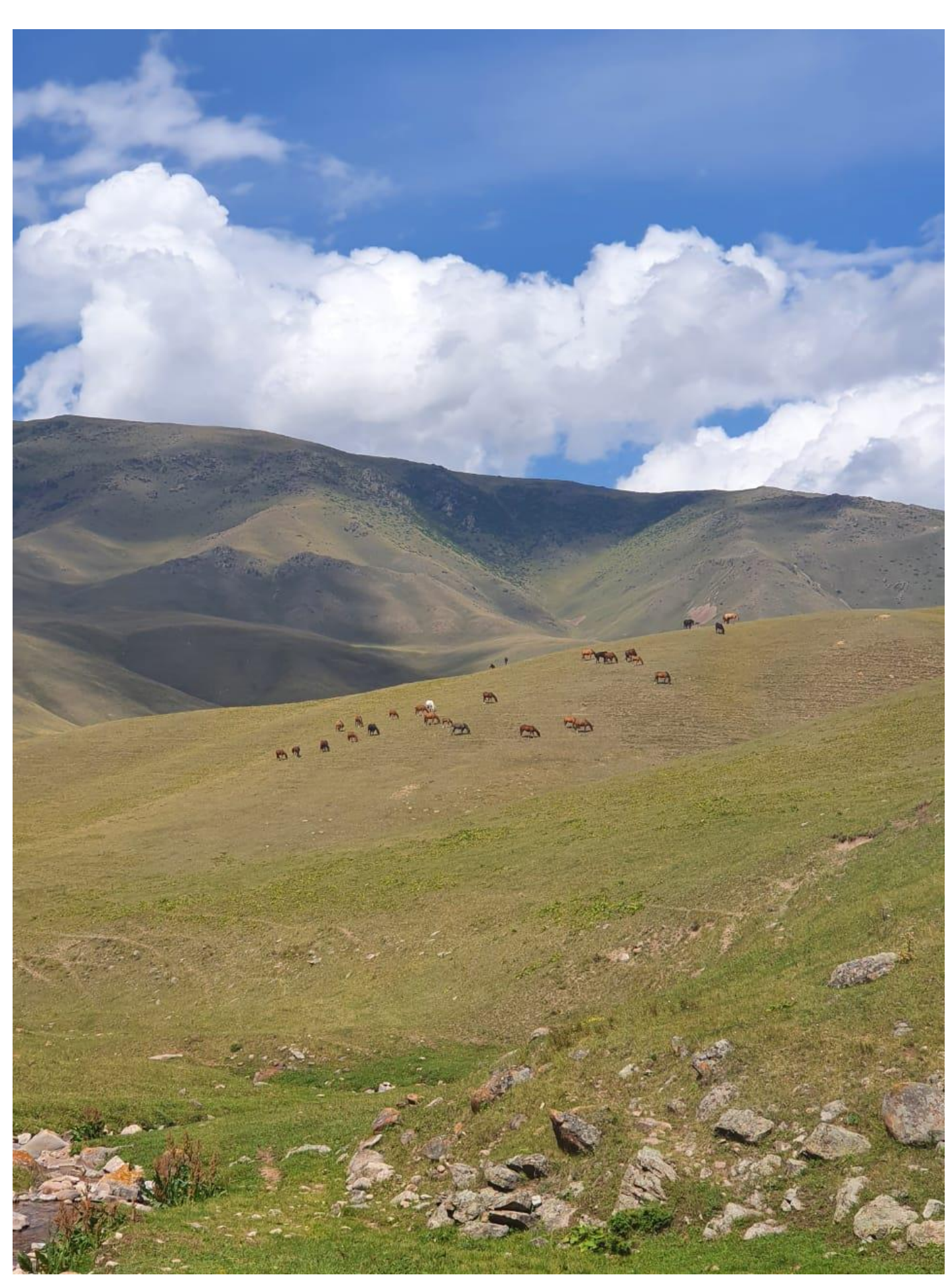

Plato Asy, Almaty region, August 2020. Botagoz Rzagazieva, Almaty, Kazakhstan. management of atrial fibrillation developed in collaboration with the European Association of CardioThoracic Surgery (EACTS). Eur Heart J 2020 Aug 29: ehaa612. 